\title{
GLAD!
}

Revue sur le langage, le genre, les sexualités

02 | 2017

Varia

\section{Les genres décrits / Les genres récrits}

Présentation de la chronique

Describing Genders / Rewriting Genders

Daniel Elmiger, Julie Abbou et Lucy Michel

\section{CpenEdition}

Journals

Édition électronique

URL : http://journals.openedition.org/glad/615

DOI : $10.4000 /$ glad. 615

ISSN : 2551-0819

Éditeur

Association GSL

Référence électronique

Daniel Elmiger, Julie Abbou et Lucy Michel, « Les genres décrits / Les genres récrits », GLAD! [En ligne], 02 | 2017, mis en ligne le 01 juin 2017, consulté le 21 janvier 2021. URL : http://

journals.openedition.org/glad/615; DOI : https://doi.org/10.4000/glad.615

Ce document a été généré automatiquement le 21 janvier 2021.

\section{(2) $\odot \Theta \Theta$}

La revue GLAD! est mise à disposition selon les termes de la Licence Creative Commons Attribution -

Pas d'Utilisation Commerciale - Pas de Modification 4.0 International. 


\title{
Les genres décrits / Les genres récrits
}

\author{
Présentation de la chronique \\ Describing Genders / Rewriting Genders \\ Daniel Elmiger, Julie Abbou et Lucy Michel
}

\section{Auteur·e·s}

1 Daniel Elmiger (pour Les genres récrits), Julie Abbou, Lucy Michel et autres personnes (pour Les genres décrits).

\section{Contenus et thématiques de la chronique}

Comment parler et écrire d'êtres humains, en français? À première vue, les deux genres grammaticaux correspondent à une binarité des sexes, mais si l'on regarde de plus près, on s'aperçoit vite que tant du point de vue de la langue que de celui des personnes désignées, les choses sont plus complexes : ainsi, le genre masculin est par exemple souvent utilisé, dans la langue, pour « inclure » le féminin (dans la grammaire traditionnelle, on dit qu'il «l'emporte») - et la correspondance entre genre grammatical et genre/sexe de la personne désignée n'est que partielle et peut se révéler pleine de surprises.

$3 \mathrm{Au}$ niveau des référents, il n'est plus légitime de simplement distinguer entre « hommes » et « femmes » - voire entre « mâles » et « femelles » : par exemple, il s'agit de distinguer entre les critères sexuels et ceux que la personne en question considère comme pertinents pour son identité de genre ou son expression de genre. En outre, le regard linguistique sur le genre s'est aussi élargi en fonction de différents cas qui défient la binarité traditionnelle des sexes/genres: on peut penser à divers types d'intersexualité, aux personnes trans*, aux identités non binaires, etc. 
4 Dans cette chronique, nous nous intéressons au genre du point de vue de sa manifestation dans la langue : le volet Les genres décrits se penche sur la codification du langage et de son fonctionnement, avec un regard critique sur les descriptions normatives et un regard éveillé sur celles qui sont alternatives qui émergent au cours du temps.

5 L'autre volet, Les genres récrits, décrit et illustre de nouvelles formes utilisées, souvent de manière expérimentale, pour parler des personnes autrement, que ce soit avec de nouveaux pronoms, des règles grammaticales réinterprétées, une morphologie expérimentale, etc.

6 Dans chaque numéro de GLAD!, l'un et/ou l'autre volet fera objet d'une chronique ; les responsables de la rubrique s'engagent à en fournir un texte et encouragent la rédaction d'un autre. L'objectif est ainsi d'avoir une chronique ou (mieux) deux par numéro de GLAD!, à propos de la description théorique des genres et des pratiques de (ré)écriture que l'on peut trouver dans des textes contemporains. Il n'est pas prévu que les deux textes se répondent du point de vue thématique - sans pourtant l'exclure.

\section{Participation}

7 Les personnes intéressées de participer à cette chronique sont invitées à s'adresser à : daniel.elmiger [at] gmail.com.

INDEX

Thèmes : Chroniques 